\title{
Gene Function
}

National Cancer Institute

\section{Source}

National Cancer Institute. Gene Function. NCI Thesaurus. Code C48933.

A term that expresses the function of a gene product. 